\title{
Cloning, expression and purification of squalene synthase from Candida tropicalis in Pichia pastoris
}

\begin{abstract}
Squalene synthase (SS) is the key precursor and first committed enzyme of the sterol biosynthesis pathway. In a previous work, SS has been identified as one of the immunogenic proteins that could be a potential diagnostic candidate for the pathogenic fungus Candida tropicalis. In this study, SS from C. tropicalis was cloned and expressed as recombinant protein in Pichia pastoris to investigate its reactivity with serum antibodies. ERG9 gene that encodes for SS was amplified by PCR and cloned in-frame into pPICZB expression vector. The recombinant construct was then transformed into P. pastoris GS115 host strain. Expression of the recombinant protein was confirmed by SDS-PAGE and Western blot analysis using anti-His tag probe. Optimal protein production was achieved by cultivating the culture with $1.0 \%$ methanol for $72 \mathrm{~h}$. The recombinant protein was purified to approximately 97\% pure in a single step immobilized metal affinity chromatography with a yield of $70.3 \%$. Besides, the purified protein exhibited specific reactivity with immune sera on Western blot. This is the first report on heterologous expression of antigenic SS from C. tropicalis in P. pastoris which can be exploited for large-scale production and further research. The results also suggested that the protein might be of great value as antigen candidate for serodiagnosis of Candida infection.
\end{abstract}

Keyword: Antigen; Candida tropicalis; Pichia pastoris; Recombinant protein; Squalene synthase 